\title{
Séismes artificiels et exploitations petrolières l'exemple de Lacq (France)
}

\author{
par J. P. Rothe $\left({ }^{*}\right)$
}

Received on October 5 th, 1977.

\begin{abstract}
Riassunto - In omaggio al professor Caloi che, dal 1956, proponeva una correlazione tra gli importanti terremoti avvenuti nel maggio 1951 nella valle del Po e lo sfuttamento dei giacimenti di gas, l'A. porta l'esempio dell'attività sismica che, dal 1969, sì è manifestata sul campo petrolifero e portatore di gas di Lacq, nel bacino di Aquitania. Vengono fornite indicazioni concernenti la complessa geologia del duplice giacimento; si noterà in particolare che, in seguito all'esistenza di una scogliera, le variazioni laterali di velocità nei diversi livellamenti sono molto importanti. Le carte macrosismiche tracciate in occasione di parecchie scosse, largamente risentite dalla popolazione, mostrano una palese relazione con il giacimento. Una rete di sismografi installata intorno al giacimento ha permesso di registrare e localizzare numerose scosse deboli. Le cause dell'attività sismica possono essere due: sfruttamento del giacimento petrolifero per mezzo di pozzi ad iniezione d'acqua, o decompressione nel giacimento di gas verso $\mathbf{i}$ $3500-4000 \mathrm{~m}$ di profondità.
\end{abstract}

RÉsumé - En hommage au Professeur Caloi qui, dès 1956, proposait une corrélation entre les importants séismes survenus en mai 1951 dans la vallée du Pô et l'exploitation de gisements de gaz l'auteur apporte l'exemple de l'activité séismique qui, depuis 1969 , s'est manifestée sur le champ pétrolifère et gazéifère de Lacq, dans le Bassin d'Aquitaine. Des indications sont données concernant la géologie complexe du double gisement; on notera en particulier que, par suite de l'existence d'un récif, les variations latérales de vitesse dans les différentes couches sont très importantes. Les cartes macroséismiques tracées à l'occasion de plusieurs secousses largement ressenties par la population montrent une relation manifeste avec le gisement. Un réseau de séismographes installé autour du gisement a permis d'enregistrer et de localiser de nombreuses secousses faibles. Les causes de l'activité séismique peuvent être doubles: exploitation du gisement pétrolifère à l'aide de puits d'injection d'eau, décompression dans le gisement de gaz vers 3500 à 4000 mètres de profondeur.

${ }^{*}$ ) Professeur honoraire à I'Université de Strasbourg; 160, rue d'Alco, 34100, Montpellier. 
L'uomo di scienza, se è veramente tale, dev'essere sempre ed esclusivamente, al servizio della verità

P. Caloi, 1970

INTRODUCTION.

Le signataire des lignes qu'on va lire se souvient encore d'avoir été intrigué par les belles inscriptions d'un séisme proche aperçues le 15 mai 1951 sur les appareils de la station séismologique de Strasbourg. Ce séisme et sa réplique du 16 mai devaient faire l'objet d'une étude détaillée de la part de P. CALo et de ses collaborateurs (P. CALo et al., 1956): la magnitude était voisine de $51 / 2$; l'épicentre $\left(45^{\circ} 18^{\prime} \mathrm{N}\right.$, $9^{0} 37^{\prime}$ E) était situé dans la plaine du Pô, non loin de Caviaga, dans une région où l'activité séismique naturelle avait toujours été faible; le foyer était peu profond $(5 \mathrm{~km})$; la secousse principale et sa réplique avaient été ressenties dans la région épicentrale avec des intensités maximales de degré VI-VII et de degré V-VI respectivement. La répartition du sens des premiers mcuvements des ondes longitudinales enregistrées dans les stations européennes présentait un caractère inhabituel contrastant avec ce qui était généralement observé dans les séismes des Alpes et des Apennins. Le séisme paraissait dû à une rupture de l'équilibre élastique, rupture qui s'était produite dans une vaste zone, particulièrement développée dans le quadrant nord-ouest, avec les caractéristiques d'une compression manifestant une violente poussée vers l'extérieur.

En conclusion à leur étude nos collègues italiens étaient amenés à formuler l'hypothèse particulièrement intéressante et hardie que la cause des séismes du 15 et du 16 mai 1951 n'était pas entièrement naturelle mais pouvait résulter d'une intervention humaine, à savoir l'exploitation d'importants gisements de gaz: dans la région même de Caviaga, épicentre des séismes, existaient des puits servant à l'extraction de quantités considérab.es de gaz méthane allant de 10000 à $300000 \mathrm{~m}^{3}$ par jour; la profondeur des couches-réservoirs variait de 1250 à $1450 \mathrm{~m}$ sous la surface et la pression maximale à la bouche des puits était de l'ordre de 130 à $140 \mathrm{~kg} / \mathrm{cm}^{2}$. L'extraction durait déjà depuis plusieurs années et la zone était donc soumise à une décompression considérable: l'extraction d'un million de $\mathrm{m}^{3}$ de gaz par jour à une pression de $100 \mathrm{~kg} / \mathrm{cm}^{2}$ doit correspondre à un travail journalier de $10^{20}$ ergs, c'est-à-dire l'énergie que l'on attribue à un séisme de magnitude 5,5; naturellement cependant la libération d'énergie doit se faıre de façon 
continue et non brusquement comme dans le cas d'un tremblement de terre.

P. Caloi (1970) a rappelé que l'explication des séismes de 1951 par l'extraction de grandes quantités de gaz dans le quadrilatère Bassiascc-Cornigliano-Ripalta-Caviaga avait été accueillie ironiquement en Italie [en particulier probablement par les exploitants!]; pourtant, en 1970, quinze ans après l'avoir formulé pour la première fois, $P$. CALor la considérait toujours comme parfaitement valable.

L'activité séismique provoquée par l'injection d'eau en profondeur, en particulier dans les exploitations pétrolières, a fait par ailleurs l'objet de nombreux travaux récents dont certains sont rapidement devenus classiques (Healy et al., 1968; Evans, 1970; McClain, 1970; Munson, 1970; Hamiltor and Meehan, 1971; Raleigh et al., 1972; Kisslinger, 1976). Au Premier Symposium international sur la séismicité induite, tenu à Banff (Canada) en 1975, MiLne et Berry (1976) ont cité un nouvel exemple, celui du séisme du 8 mars 1970, de magnitude (mB) 5,1 , qui s'est produit dans le champ pétrolifère de Snipe Lake où d'importantes injections d'eaux avaient eu lieu et où on ne connaissait pas jusqu'alors d'activité séismique; il semble que ce soit d'ailleurs le seul cas connu au Canada d'un séisme probablement provoqué par l'injection d'eau dans un champ pétrolifère en exploitation.

Au même symposium Yerkes et Castle (1976) ont, de leur côté, rappelé que la séismicité superficielle, accompagnée parfois de la formation de fractures et de failles, attribuable à la seule extraction de fluides dans les exploitations pétrolières est moins bien connue mais probablement aussi répandue que la séismicité en relation avec les injections de fluides. Ces auteurs ont donné des détails sur deux exemples observés aux Etats-Unis concernant les champs pétrolifères de Goose Creek (Texas) et de Wilmington (Californie). Plusieurs autres cas moins caractéristiques ont également été cités. Dans les exemples étudiés l'extraction de fluide peut avoir été dans une deuxième phase accompagnée d'injections et les effets relatifs de l'extraction de fluide seule et des injections ne sont pas faciles à séparer.

On rappelera encore la communication présentée au cours de l'Assemblée générale de l'U.G.G. I. tenue à Grenoble en 1975 et intitulée "Essaim de séismes causés par une exploitation intensive d'un champ pétrolifère" (Smirnova, Navitzkaya et Shebalin, 1975). Le réservoir, dans le champ pétrolifère de Grozny, au nord du Caucase, est situé dans des calcaires fissurés du Crétacé supérieur, dans des conditions 
géologiques très semblables à celles du réservoir de Lacq-supérieur dont il sera question plus loin; au cours des quatre années d'exploitation 5 millions de tonnes d'huile ont été extraites et la pression du gisement soviétique a baissé de 100 atmosphères. Une injection d'eau $\left(550000 \mathrm{~m}^{3}\right)$ s'est accompagnée d'une augmentation brusque de l'activité séismique sous forme d'un essaim de secousses dont la magnitude a atteint le chiffre 4; une intensité maximale de degré VII a élé observée; la carte macroséismique fait apparaître une forte intensité épicentrale pour une surface macroséismique relativement faible. Un graphique montre une corrélation très nette entre les quantités d'eau injectée et l'activité séismique.

L'exemple de Laco (France).

Structure ciu double gisement pétrolifère et gazéifère.

Le gisement pétrolifère et gazéifère de Lacq, à $25 \mathrm{~km}$ au nord-ouest de Pau, préfecture du département des Pyrénées-Atlantiques, est situé dans le Bassin d'Aquitaine, en bordure nord des Pyrénées, dans une région où l'épaisseur des terrains sédimentaires dépasse 10000 mètres.

La structure de Lacq a été découverte en 1949 par prospectioli séismique. Au point de vue tectonique les marnes yprésiennes reposent directement sur les calcaires campaniens; l'absence de crétacé supérieur (Danien, Maestrichtien et une partie du Campanien) démontre que la structure a dû s'ériger entre le Campanien et l'Yprésien et subir une longue période d'émersion. Les géologues pensaient découvrir à Lacq des réservoirs sous une épaisse couche de "Flysch »; l'hypothèse s'est rćvélée fausse, par contre les forages ont permis de découvrir un Campanien-Santonien qui, bien que calcaire, est productif de pétrole grâce à sa fissuration et à sa dolomitisation.

Ce gisement, dit de Lacq-supérieur, a été exploité à partir de 1950; il a la forme d'une ellipse d'une surface totale de $6,8 \mathrm{~km}^{2}$; une cinquantaine de puits on été forés; leur profondeur moyenne est de $650 \mathrm{~m}$; 5 puits demeurent éruptifs, 24 produisent par pompage. La production a atteint 300000 tonnes en 1954; elle est actuellement de l'order de 50000 tonnes par an; la production cumuiée a dépassé j millions de tonnes. Parmi les puits anciennement productifs 5 ont été 
utilisés pour la réinjection d'eau. La pression du gisement a peu varié par rapport à la pression initiale, ce qui peut s'expliquer - pensent les ingénieurs de la Société - par le fait que le pétrole surmonte une vaste nappe acquifère contenue dans un énorme massif calcaire et dolomitique.

Des forages profends, placés sur le sommet de la structure, ont permis de découvrir en 1951 le gisement dit de Lacq-profond. La structure, elliptique, est beaucoup plus importante que celle de Lacqsupérieur: le grand axe mesure environ $15 \mathrm{~km}$, le petit axe $8 \mathrm{~km}$; la superficie du réservoir producteur est évaluée à $75 \mathrm{~km}^{2}$. Il s'agit d'un gisement de gaz humide à forte teneur de soufre $\left(15,3 \%\right.$ de $\left.\mathrm{SH}^{2}\right)$. Le réservoir est constitué par le Néocomien et le Purbeckien (sommet du Jurassique), à une profondeur variant entre 3500 et 5200 mètres; 34 puits on été forés jusqu'au réservoir gazéifère; 27 sont en exploitation. La production est actuellement de l'ordre de 7,5 milliards de $\mathrm{m}^{3}$ par an; la production cumulée, depuis la mise en exploitation en 1955, a dépassé 130 milliards de $\mathrm{m}^{3}$.

La coupe du forage Lacq 103 est donnée ci-dessous:

mètres

0-15 Alluvions

15-260 Mollasse oligo-miocène

260-500 Marnes yprèsiennes

500-544 Brèche marno-calcaire paléocène

544-630 Calcaire marneux campanien

630-800 Sénonien: gisement de pétrole (Lacq-supérieur)

800-985 Dolomie (Turonien-Cénomanien)

985-1430 Calcaire dolomitique albien-aptien

1430-2830 Dolomies et calcaires récifaux (Aptien)

2830-3460 Marnes de Sainte-Suzanne (Aptien)

3460-3550 Aptien calcaire dolomitique

Néocomien (mauvais réservoir)

Purbeckien calcaire: principal réservoir de gaz (gisement de Lacq-projond.

Une coupe de la structure de Lacq est reproduite Fig. 1. 


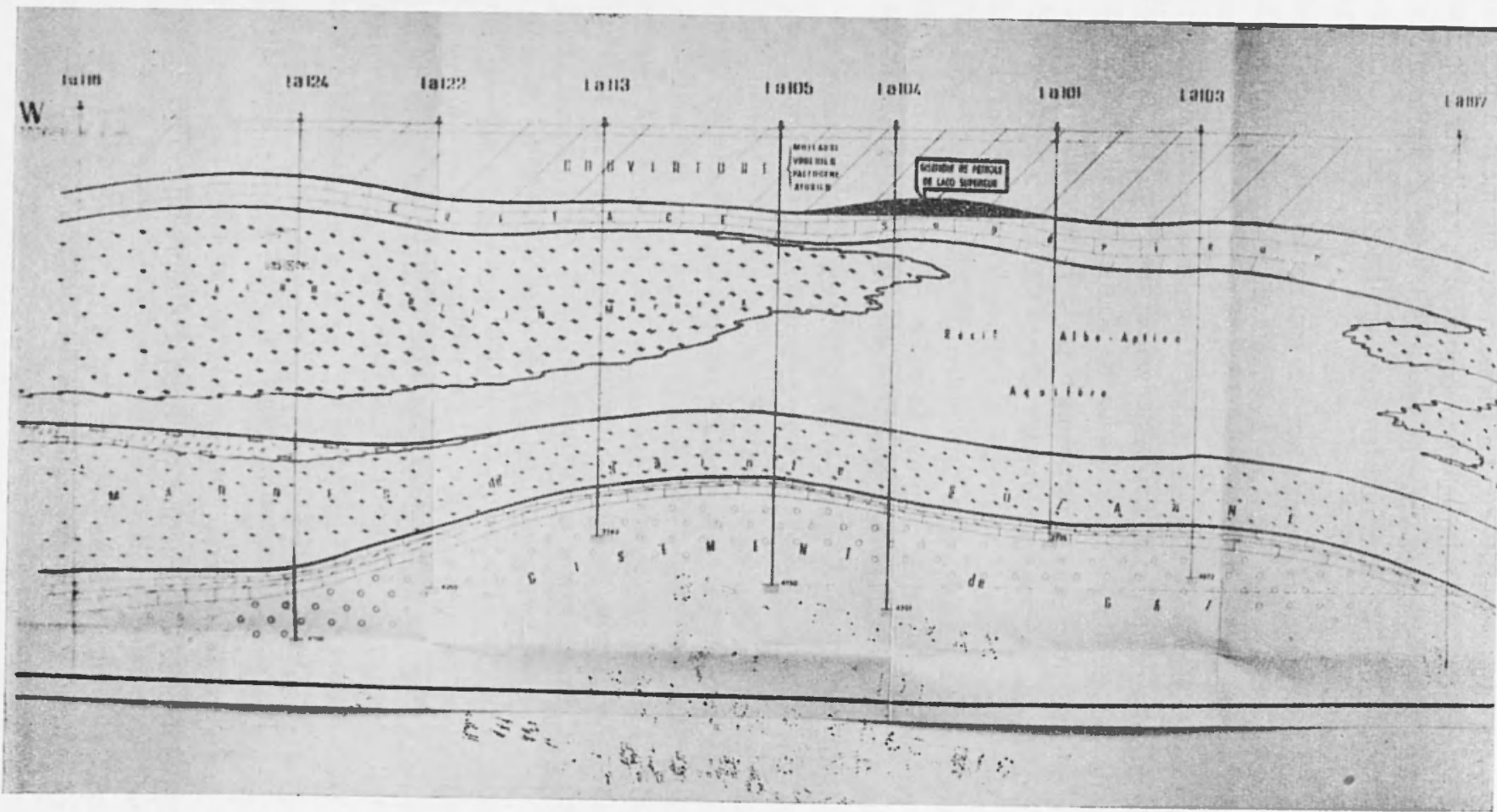

Fig. 1. Coupe Ouest-Est de la structure de Lacq (Document S. N.P. A.). 
L'activité séismique sur le double gisement.

La structure de Lacq est située à une trentaine de kilomètres au Nord du "front nord-pyrénéen », zone séismique particulièrement active; de nombreux séismes originaires de cette zone sont ressentis plus ou moins largement dans le Bassin d'Aquitaine mais jusqu'en 1969 aucun épicentre netait connu dans la région même de Lacq, comme d'ailleurs dans les autres parties de la province séismotectonique aséismique constituée par le Bassin d'Aquitaine.

Un premier séisme, enregistré dans 36 observatoires, s'est produit le 24 novembre 1969 à $10 \mathrm{~h} 51 \mathrm{mn} 50 \mathrm{~s}$. Ce séisme est décrit dans mon étude sur la séismicité de la France pendant la décennie 1961-1970 (J. P. RотнE, 1972). Une détermination épicentrale provisoire ayant indiqué la région de Lacq, une large enquête macroséismique par questionnaires fut effectuée: 357 réponses en provenance de localités différentes ont permis de dresser la carte reproduite fig. 2; la surface

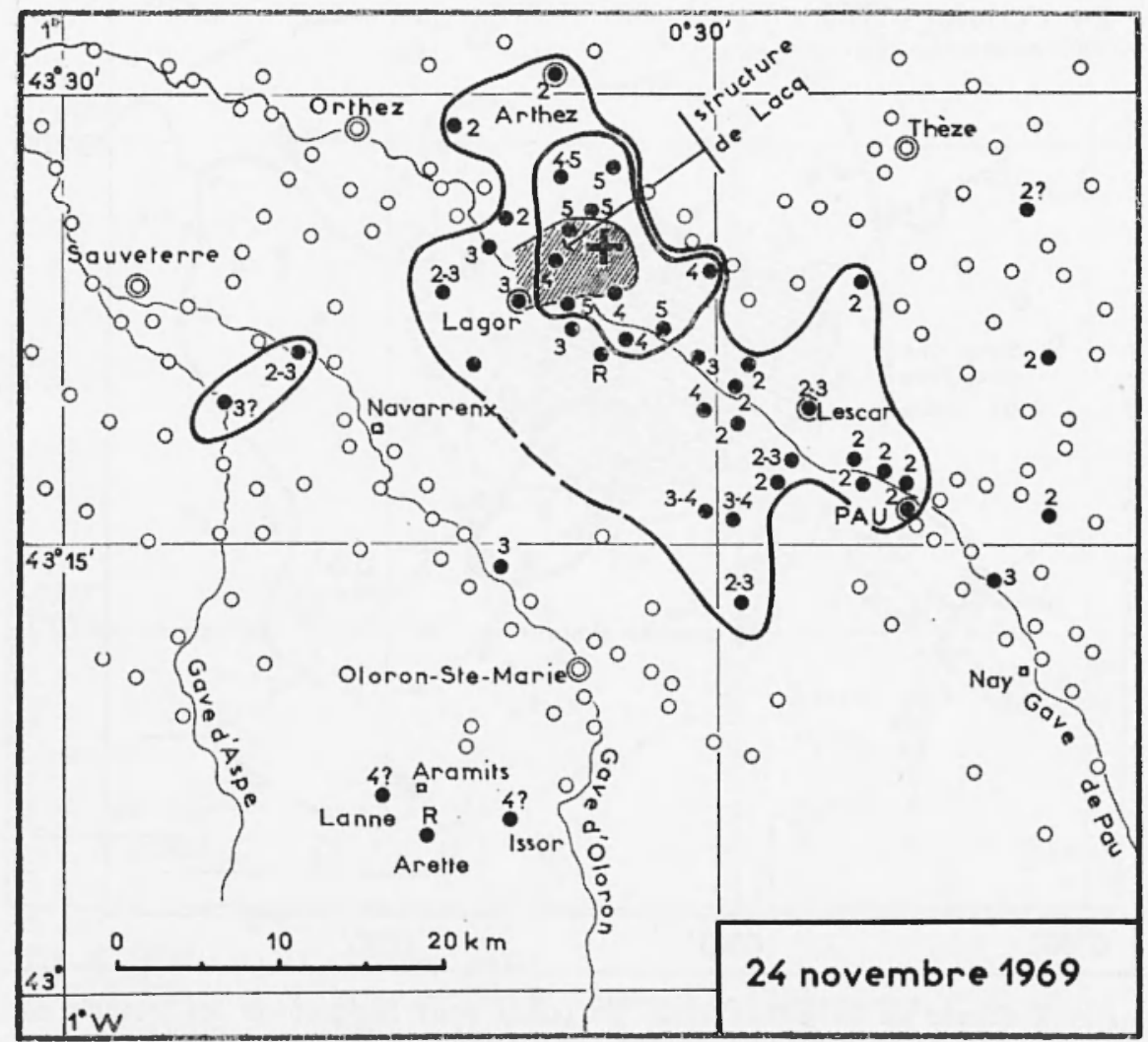

Fig. 2. Séisme du 24 novembre 1969: Isoséistes; les points norrs indiquement les localités où la secousse a été ressentie. 
macroséismique mesurait environ $700 \mathrm{~km}^{2}$; parmi les 47 réponses positives 'lintensité $V$ était signalée à Urdès, Castil!on, Audéjos, Labastide-Cézéracq et Os-Marsillon, l'intensité IV-V à Arthez, l'intensité IV à Arbus, Artix, Besingrand, Cescau et Lacq. La zone d'intensité maximale correspondait exactement avec la structure de Lacq; la secousse avait été ressentie en direction sud-est jusqu'à Pau, en particulier dans les villages situés sur les alluvions du Gave de Pau: à l'examen de cette carte j'avais, dès 1969, cherché une relation entre ce séisme et l'exploitation du gisement.

Le 14 décembre 1971, à $22 \mathrm{~h} 21 \mathrm{mn} 24 \mathrm{~s}$, une nouvelle secousse était enregistrée dans 27 stations; l'épicentre calculé $\left(43^{\circ} 18^{\prime} \mathrm{N}, 0^{\circ} 36^{\prime} \mathrm{W}\right)$ était encore situé dans la région de Lacq; cependant l'enquête macrosćismique faite très tardivement n'a pas fourni de résultats positifs.

Le 24 janvier 1972, à $02 \mathrm{~h} 47 \mathrm{mn}$, une secousse est ressentie dans la partie sud-est du gisement de Lacq: on note l'intensité IV à Besingrand et à Abos, l'intensité III à Tarsacq, Noguères, Pardies, Lacommande

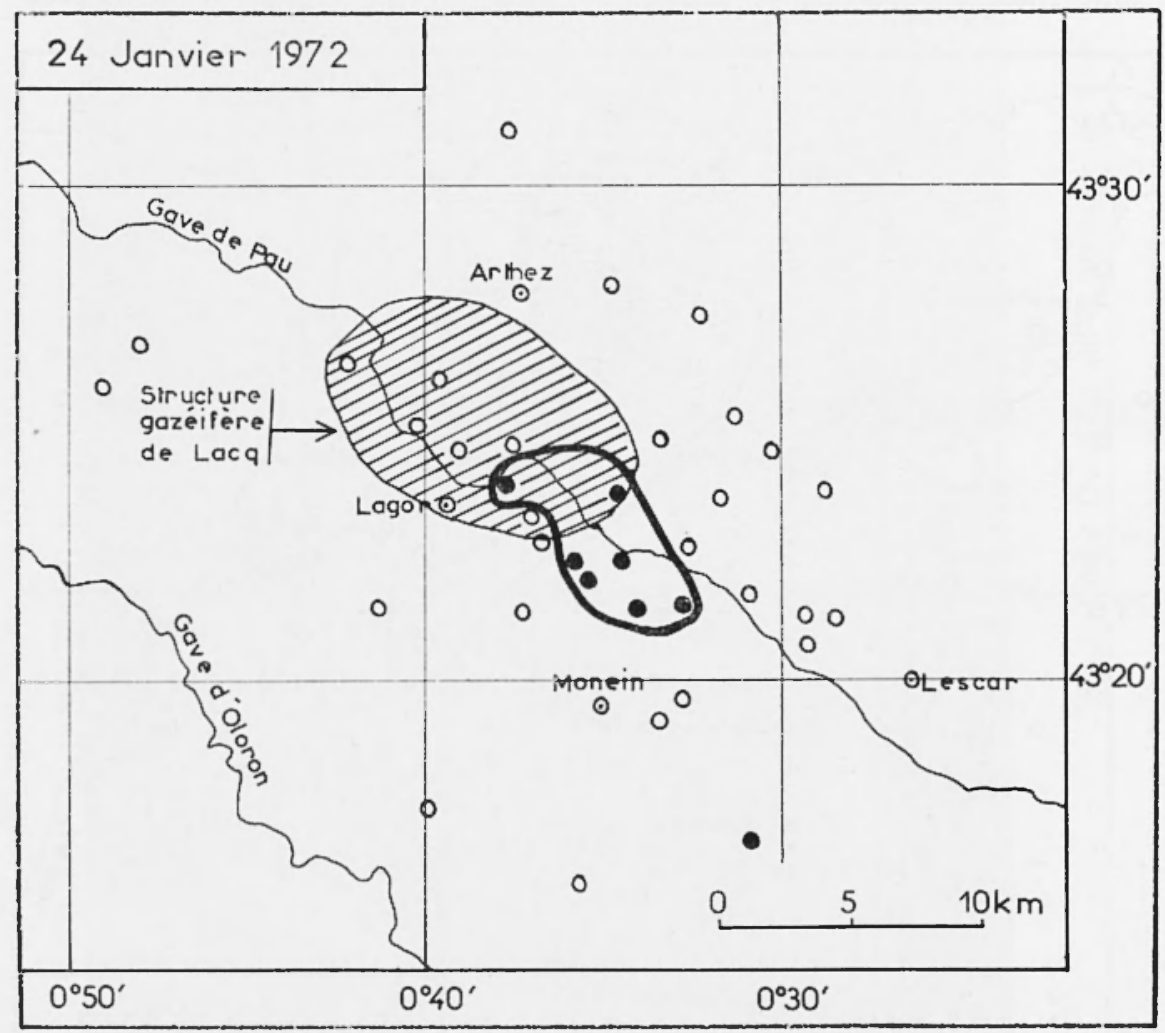

Fig. 3. Sćisme du 24 janvier 1972: les points noirs indiquement les localités où la secousse a été ressentie. 
et Artix (fig. 3). Entre le 24 janvier et le 2 février 1972 une dizaine d'autres secousses très localisées sont ressenties dans la même région inquiétant la population et attirant l'attention de la Drrection départementa'e de la Protection Civile.

La plus importante des secousses ressenties par la population s'est produite le 31 décembre 1972 à $07 \mathrm{~h} 16 \mathrm{mn} 56 \mathrm{~s}$. L'intensité $V$ a été átteinte à Abidos, Arance, Audéjos, Lâa-Mondrans, Lacq, Lagor, Mont, Mourenx, Sauvelade et Vielleségure, l'intensité IV-V à Artix, l'intensité IV à Besingrand, localités situées sur ou à proximité du gisement. Le rayon mcyen de la surface macroséismique était compris entre 15 et $20 \mathrm{~km}$; la secousse a été inscrite jusqu'à $1200 \mathrm{~km}$ de distance par une trentaine de stations séismologiques; le calcul à l'ordinateur a fourni un épicentre $\left(43^{0} 25^{\prime} \mathrm{N}, 0^{n} 38^{\prime} \mathrm{W}\right)$ pratiquement confondu avec l'épicentre macroséismique (fig. 4).

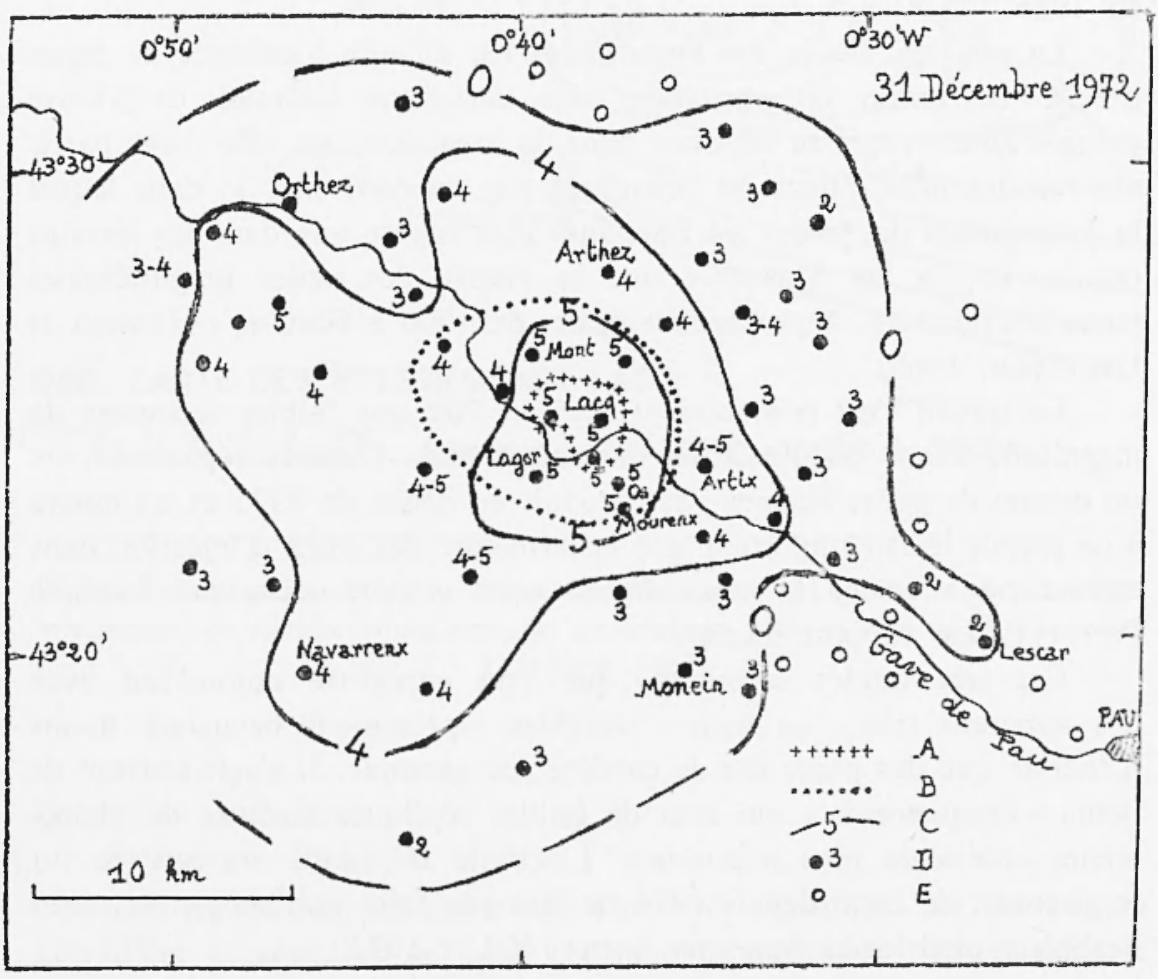

Fig. 4. Séisme du 31 décembre 1972:

$A$ : Gisement de Lacq-supérieur; $B$ : Gisement de Lacq-profond; $C$ : Isoséistes; $D$ : secousse ressentie et intensité; $E$ : Secousse non ressentie. 
Quelques semaines avant cette forte secousse j'avais eu des entretiens avec le Directeur de la Société qui exploitait le gisement, la Société nationale des Pétroles d'Aquitaine (S. N.P.A.), devenue depuis la Scciété Elf-Aquitaine (S. N.E. A.). 11 fut décidé d'installer un réseau de 4 station séismologiques reliées par télétransmission à une station centrale située à l'usine de Lacq.

Le réseau est devenu opérationnel en 1974. Au cours des 6 premiers mois d'enregistrement 86 chocs, tous faibles, ont été inscrits dont 42 sont des séismes extérieurs au champ pétrolifère, séismes naturels localisés sur le "front nord-pyrénéen " ou séismes artificiels (explosions dans des carrières en particulier). Les 44 autres secousses sont situées sur ou au voisinage de la structure de Lacq: 18 au sud-ouest du village de Lacq, 6 au voisinage du village d'Artix (foyer des secousses de janvier 1972), 6 dans la partie nord du gisement, 7 dans la région de Monein, au sud, à proximité du nouveau gisement de gaz de Pau-Meillon découvert en 1966.

La position exacte des hypocentres est difficile à calculer de façon précise en raison en particulier des variations latérales de vitesse considérables que l'on observe dans la structure; en effet une partie des terrains albo-aptiens est constituée par un récif (fig. 1) dans lequel la propagation des ondes est beaucoup plus rapide que dans les terrains encaissants; la fig. 5 montre que la vitesse des ondes longitudinales passe latéralement, à profondeur égale, de 4000 à $6000 \mathrm{~m} / \mathrm{s}$ (JENNER et Dienesch, 1965).

Le travail s'est poursuivi et environ 200 très faibles secousses de magnitude allant jusqu'à 2 ont été enregistrées. Dans la région d'Artix un essaim de petits séismes s'est produit au début de 1975 et on notera à ce propos le fait important que la fermeture des puits d'injection dans cette zone a amené la cessation de cette activité séismique localisée (WITTLINGER, rapport inédit).

Ces très faibles secousses, que l'on enregistre aujoud'hui avec des appereils très - ou trop - sensibles représentent beaucoup moins d'énergie que des petits tirs de carrière par exemple. Il s'agit souvent de petits «craquements » qui sont de faibles répliques tardives de phénomènes antérieurs plus importants. L'activité séismique entregistrée sur le gisement de Lacq depuis 1974 ne doit pas faire oublier les secousses beaucoup plus fortes ressenties entre 1969 et 1972 . 


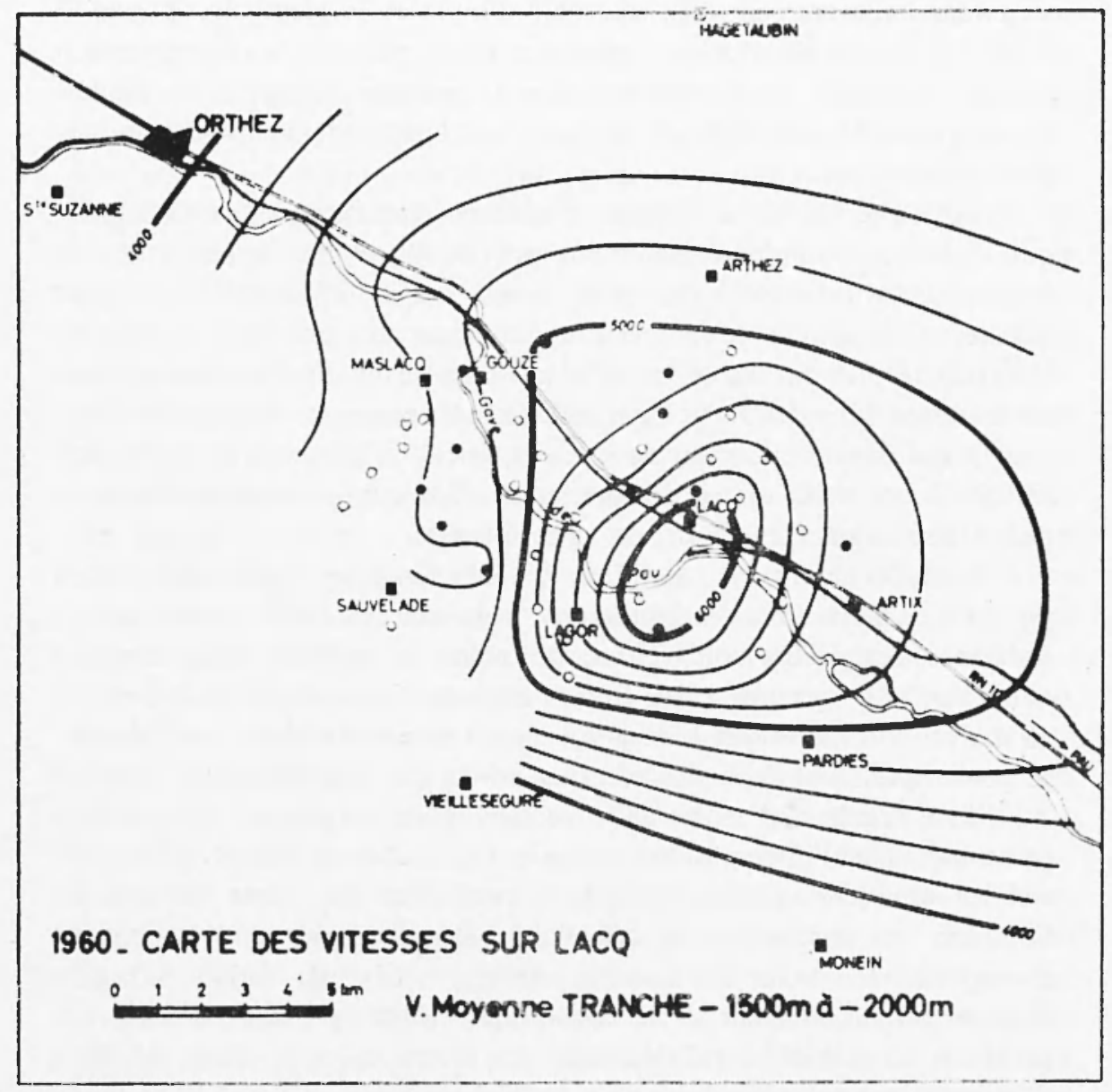

Fig. 5. Variation latérale de la vitesse dans la structure anticlinale de Lacq dans une tranche de terrains situés entre les prolondeurs 1500 et $2000 \mathrm{~m}$ (P. Jenner et I. DIENESCH, 1965).

\section{Quelques remarques.}

L'interprétation complète de l'activité séismique sur le champ pétrolifère et gazéifère de Lacq est encore inachevée; elle est délicate en raison même de la complexité du double gisement, de la variation latérale des vitesses dans la structure, de l'existence d'un réservoir 
acquifère important entre le réservoir d'huile et le réservoir de gaz. Il est difficile aussi de séparer l'influence, d'une part de la décompression qui doit résulter de l'extraction d'une quantité de gaz qui dépasse aujourd'hui 130 milliards de $\mathrm{m}^{3}$, et d'autre part de l'exploitation par puits d'injection.

MCCLAin (1970) a indiqué plusieurs conditions nécessaires pour que l'injection de fluide entraîne une activité séismique. Le réservoir doit avoir une porosité suffisante mais une perméabilité assez basse pour qu'une augmentation de la pression des pores soit possible: le type de réservoir le plus favorable est celui où la porosité résulte d'un système de fractures dans des roches par ail'eurs non poreuses et imperméables: c'est le cas dans le gisement de Lacq-supérieur. L'injection de fluide doit se faire à une échelle et à des pressions telles que la pression des pores puisse être augmentée dans une vaste étendue.

Pour MCClain la condition de loin la plus importante est la présence d'une tension tectonique régionale de telle sorte que la résistance des roches soit voisine du point de rupture; cela implique que les puits d'injection soient situés dans une région d'activité tectonique au moins modérée et que le réservoir soit à une profondeur considérable. Je pense qu'il faut faire des réserves en ce qui concerne cette dernière condition, également souvent invoquée pour expliquer les séismes provoqués par l'intervention humaine (P. CALOI, 1970, p. 292); on cas les exemp'es connus intéressent aussi bien les vieux terrains où l'orogénie est ancienne et la séismicité naturelle faible que les régions d'orcgénie récente et de notable séismicité (RothÉ, 1970). C'est la rupture par cisaillement de la roche sous l'effet de l'augmentation des pressions interstitielles qui déclanche les secousses et B. DiAB (1976) a démontré au moyen d'un exemple numérique et en utilisant la méthode des éléments finis que la diminution de l'énergie de déformation d'un massif rocheux à la suite de la rupture des joints de discontinuité peut être équivalente à l'énergie libérée par un séisme de magnitude 6 . A cet égard P. CALor a sans doute fait preuve de beaucoup d'optimisme en limitant au chiffre 4 la magnitude maximale des séismes artificiels provoqués par lintervention humaine (P. CALOI, 1970, p. 292); on sait aujourd'hui que dans plusieurs cas la magnitude 6 a pu être atteinte ou dépassée (réservoirs de Hsinfengkiang, Kariba, Kremasta, Koyna).

En ce qui concerne l'exemple de Lacq il est acquis que l'exploitation du double gisement s'est accompagnée d'une activité céismique indis- 
cutable, bien mise en évidence par les observations macroséismiques, activité qui a passé par un maximum entre 1969 et 1972, periode pendant laquelle plusieurs secousses on été ressenties par la population; cette activité se poursuit actueilement, mais plus faiblement. Cependant je n'ai pu jusqu' ici avoir communication des dcnnées techniques indispensables à une interprétation cohérente de cette séismicité; il serait en particulier nécessaire de connaître, d'une part la chronologie exacte de la mise en fonctionnement des puits d'injection, et d'autre part la position exacte des foyers des chocs enregistrés ainsi que la précision avec laquelle ces foyers sont déterminés.

Dans son courageux article déjà cité P. CaLOI (1970) a montré combien il est difficile de faire admettre des conclusions qui vont à l'encontre de certains intérêts économiques ou politiques. Les Sociétés ont parfois tendance à éviter de reconnaitre leurs responsabilités dans des phénomènes qui pourraient être dangereux et qui résultent d'une intervention humaine rompant un équilibre naturel beaucoup plus fragile qu'cn ne le pense généralement. P. CaLor a donné des exemples tirés de son expérience dans son propre pays; il a rappelé aussi qu'aux Etats-Unis c'est seulement après d'âpres controverses qu' Evans a réussi - partiellement - à convaincre les autorités de Denver que les séismes qui secouaient fortement la ville et les environs étaient dûs à l'injection d'eux uséees dans le puits de l'Arsenal; Evans écrivait à CALOI en 1968 que les autorités avaient tout fait pour éluder leurs responsabilités ( .... although the political authorities are doing the best to dodge the responsabilities ...»). En France les relations qui pouvaient exister entre le remplissage de certains lacs-barrages et les séismes qui se sont produits à proximité n'ont pas non plus été facilement reconnues par les autorités; il est vrai que ces séismes avaient fait tomber des cheminées et lézardé des murs, la question des responsabilités pouvait donc se poser.

Si certaines Sociétés veulent bien engager des moyens importants et des experts pour étudier des phénomènes encore mal compris les résultats de ces études restent trop souvent confidentiels et sont perdus pour la Science. 


\section{BIBLIOGRAPHIE}

ANONYME, 1972, - Note technique sur les gisements de Lacq supérieur et de Lacq profond. S. N.P. A. (Division Production), Pau, 4 p., 4 fig. hors-texte.

Caloi P., De Panfilis, M., Di Filippo D., Marcelli L., Spadea M. C., 1956. - Terremoti della Val Padana del 15-16 maggio 1951. Annali di Geofisica, Roma, IX, pp. 65-105.

CAlor P., 1970. - How nature reacts on human intervention; responsabilities of those who cause and who interpret such reactions. Annali di Geofisica, Roma, XXIII, pp. 283-305.

Dıнв B., 1976. - Une explication de l'origine de l'énergie libérée lors des séismes induits par le remplissage de certains lacs-barrages. Engineering Geology, 10, pp. 283-292.

Evans D. M., 1970. - The Denver Area Earthquakes and the Rocky Mountain Arsenal Disposal Well. Engineering Geology, Case Histories $\mathrm{Nr} 8$. Geolog. Soc. Amer., I, pp. 25-32.

Hamilton D. H. and Melhan R. L., 1971. - Ground rupture in the Baldwin Hills, Science, 172, pp. 333-344.

Healy J. H., Rubey W. W., Griggs D. F. and Raleigh C. B., 1968. - The Denver Earlhquakes. Science, 161, pp. 1301-1310.

Jenner P. et Dienesch J., 1965. - Histoire géophysique du champ de Lacq. Geophysical Prospecting, XIII, pp. 518-540.

KISSLINGER C., 1976. - A review of theories of mechanisms of induced seismicity. Engineering Geology, 10, pp. 85-98.

MCCLAIN W. C., 1970. - On earthquake produced by underground fluid injection. Oak Ridge Natl. Lab., Tenn., ORNL-TM-3154.

Milne W. G., 1976. - Induced seismicity in Canada. Engineering Geology, 10, pp. 219-226.

Munson R. C., 1970. - Relationship of Effect of Waterflooding of the Rangely Oil Field, Engineering Geology Case Histories, $\mathrm{Nr}$ 8. Geolog. soc. Amer., 1, pp. 39-49.

Raleigh C. B., Healy J. H. and Bredehoeft J. D., 1972. - Faulling and crustal stress at Rangely, Colorado. In: Flow and fracture of rocks (Gcophys. Monog. 16), Am. Geopliys. Union, pp. 275-284.

RotнÉ J. P., 1970. - Séismes artificiels. Tectonophysics, 9, pp. 215-238.

RotHÉ I. P., 1972. - La séismicité de la France de 1961 à 1970. Annales Inst. Phys. Globe Strasbourg, 3ème partie (Géophysique). IX, Strasbourg, pp. 3-134. 
Smirnova M. N., Navitzkaya N. A. and Shebalin N. V., 1975. - Earthquake Swarm caused by intensive exploitation of an oil field. XVIeme General Assembly 1. U. G. G., I. A. S. P. E. I., Abstracts, p. 39.

Yerkes R. F. and CASTLE R. O., 1976. - Seismicity and Faulting attributable to Fluid Extraction. Engineering Geology, 10, pp. 151-167. 